\title{
DIRIGER LE SPORT INTERNATIONAL : BIOGRAPHIES, TRAJECTOIRES ET RÉSEAUX D'UNE ÉLITE DU SPORT INTERNATIONAL (1920-1980)
}

\author{
Grégory Quin et Cyril Polycarpe
}

De Boeck Supérieur | «Staps »

2019/3 n $125 \mid$ pages 7 à 13

ISSN 0247-106X

ISBN 9782807393394

Article disponible en ligne à l'adresse :

https://www.cairn.info/revue-staps-2019-3-page-7.htm

Distribution électronique Cairn.info pour De Boeck Supérieur.

(C) De Boeck Supérieur. Tous droits réservés pour tous pays.

La reproduction ou représentation de cet article, notamment par photocopie, n'est autorisée que dans les limites des conditions générales d'utilisation du site ou, le cas échéant, des conditions générales de la licence souscrite par votre établissement. Toute autre reproduction ou représentation, en tout ou partie, sous quelque forme et de quelque manière que ce soit, est interdite sauf accord préalable et écrit de l'éditeur, en dehors des cas prévus par la législation en vigueur en France. Il est précisé que son stockage dans une base de données est également interdit. 


\title{
Diriger le sport international : Biographies, trajectoires et réseaux d'une élite du sport international (1920-1980)
}

\section{Leading international sport: Biographies, trajectories, and networks of an elite in international sport (1920-1980)}

\author{
Grégory QUIN \\ Institut des Sciences du Sport de l'Université de Lausanne \\ Université de Lausanne \\ Quartier Unicentre \\ Bâtiment Synathlon \\ CH- 1015 Lausanne \\ gregory.quin@unil.ch \\ Cyril POLYCARPE \\ MCF - INSPÊ \\ Laboratoire C3S EA 4660 \\ Université de Bourgogne-Franche-Comté \\ cyril.polycarpe@univ-fcomte.fr
}

Président de la Fédération Internationale de Ski (FIS) depuis 1998, Gian-Franco Kasper est un exemple emblématique d'une trajectoire d'un dirigeant international du sport, à la fois pour les réseaux qu'il incarne et par sa trajectoire individuelle. ${ }^{1}$ En 1998, il succède à Marc Hodler, un autre dirigeant suisse, après avoir occupé la fonction de secrétaire général de l'institution entre 1979 et 1998, et rejoint en 2000 le Comité International Olympique (CIO), où il contribue à l'organisation des Jeux olympiques d'hiver de 2002, 2006 et 2010. Formé dans un établissement privé des Grisons (le Lyceum Alpinum de Zuoz) et au journalisme à l'Université de Zurich, il peut très précocement s'appuyer sur les réseaux de son père - Peter Kasper, directeur de l'Office du tourisme de Saint-Moritz entre les années 1940 et les années 1970. Il obtient ainsi son premier emploi au Courrier de Saint-Moritz et plus tard, en 1974, il participe à la création d'un office du tourisme suisse à Montréal. Actif dans les milieux du ski et du tourisme, il s'appuie également sur les réseaux construits par Marc Hodler à la FIS - qu'il préside depuis 1951
- et plus largement sur la scène internationale sportive, comme le révèle la contribution de Sébastien Cala et de Grégory Quin dans le présent numéro spécial.

Si Gian-Franco Kasper a récemment été critiqué pour ses prises de position à l'encontre des mouvements écologistes et des entraves qu'ils peuvent faire peser sur l'organisation de compétitions sportives dans les États démocratiques, il ne nous appartient pas de juger des affaires en cours ni de nous substituer au discours journalistique. Cependant, nous pouvons souligner que ces réseaux ne sont pas nouveaux en substance et que l'essor même du sport, entraînant un développement des enjeux pesant sur lui (politiques, culturels, économiques, diplomatiques, etc.) tout au long du $\mathrm{XX}^{\mathrm{e}}$ siècle, comme l'illustrent les contributions d'un ouvrage récemment paru (Quin, Vonnard \& Jaccoud, 2019). Dans l'un des derniers numéros spéciaux de la revue Staps, sur "Les femmes, actrices de l'EPS » (Szerdahelyi \& Robène, 2019), plusieurs contributions révèlent combien il est pertinent et stimulant d'envisager d'analyser l'histoire du

1 Nous sommes reconnaissant à Mme Shani D'Cruze pour sa relecture des versions anglaises des contributions de ce numéro spécial. 
sport et de l'éducation physique au prisme des biographies et des réseaux de relations (et/ ou d'influences) qui se développent entre les individus.

Dans le sillage du renouveau récent de la réflexion sur l'enquête biographique comme outil de connaissance historique (par exemple : Lemercier \& Picard, 2012 ; Milza, 2012), notre ambition est, par le biais de quelques exemples sur différent·e.s dirigeant.e.s du sport international, de poser de premiers jalons vers une prosopographie des acteurs du champ sportif international. Dans ce cadre, les contributions ici rassemblées s'ancrent dans une période clé qui s'étend depuis les premières décennies du $\mathrm{XX}^{\mathrm{e}}$ siècle, moment qui correspond à l'intensification de l'internationalisation du sport (Keys, 2006), jusqu'aux années 1980, période qui correspond à l'affirmation de sa globalisation. À ce moment, les dirigeants, s'ils sont encore pour la plupart amateurs et issus des domaines variés, vont devenir de véritables managers professionnels du sport ayant suivi un cursus de formation en gestion, droit ou économie (Bayle \& Clastres, 2018). Dans ce contexte, le sport va être profondément influencé par des dynamiques sociopolitiques comme l'industrialisation, l'impérialisme ou encore les nationalismes. De fait, le recours aux biographies nous permet alors ici de nous interroger de manière singulière sur la réactualisation tout au long du $\mathrm{XX}^{\mathrm{e}}$ siècle de ces influences dans les organisations sportives ou autour des compétitions internationales.

De fait, dans le cadre de ce numéro spécial, notre objectif est de rassembler des contributions décrivant l'engagement d'une vingtaine d'individus qui ont participé à l'établissement, puis au développement des sports au niveau international tout au long du siècle. Surtout, il convient de souligner que les études sur ces individus sont encore rares. Certes, dans le sillage d'études réalisées sur Pierre de Coubertin (en particulier : MacAloon, 2008 ; Clastres, 2011), quelques autres dirigeants internationaux ont été l'objet de réflexions.
Depuis une vingtaine d'années, la Fédération Internationale de Football Association (FIFA) a bénéficié d'attention particulière autour de ses présidents (Tomlinson, 2000 ; Quin \& Vonnard, 2018), ou d'autres acteurs, comme Ernst Thommen (Homburg, 2008 ; Vonnard, 2019). Cependant, ces réflexions sont souvent isolées et ne permettent pas de dégager de possibles convergences et divergences entre les biographies étudiées. Des études allemandes ont cependant ciblé des acteurs du développement du football international durant l'entre-deuxguerres (Peiffer \& Schulze Marmeling, 2008). Soulignant le rôle de la Suisse dans la diplomatie sportive, des contributions mentionnent le rôle et la place de dirigeants helvètes dans le développement d'organisations internationales sportives (Quin, Vonnard \& Jaccoud, 2019). Des historiens anglo-saxons privilégient quant à eux les dynamiques de l'héroïsation entourant les sportifs ou les acteurs du jeu (Carter, 2006 ; Vramplew, 2016 ; Dyer \& Day, 2017), même si celles-ci sont davantage focalisées sur le niveau national. À ce jour, l'ouvrage le plus ambitieux à l'échelle internationale est sans doute le livre collectif interdisciplinaire codirigé par Emmanuel Bayle et Patrick Clastres (2018), qui offre de précieuses informations sur plusieurs dirigeants ayant œuvré au sein de différentes organisations internationales aux $\mathrm{XX}^{\mathrm{e}}$ et $\mathrm{XXI}^{\mathrm{e}}$ siècles.

Influencé par ces travaux, un groupe de jeunes chercheurs a organisé deux conférences dans le cadre du Réseau d'études des relations internationales sportives (RERIS) à Barcelone, respectivement en juillet 2016 et 2017, avec pour thématique les dirigeants du sport international. En complément d'un ouvrage collectif spécifiquement consacré aux trajectoires de diverses dirigeantes (Nicolas \& Cervin, sous presse), ce numéro spécial est composé de plusieurs contributions présentées lors de ces deux manifestations scientifiques. Loin de considérer le genre biographique comme un objet en soi, notre exploration se propose d'éclairer à la fois les processus de la genèse 
de l'institutionnalisation des sports (modalités de gouvernance des institutions, créations de compétitions, etc.) et les façons multiples dont les processus de prises de décision s'intègrent dans l'existence individuelle et dans la vie collective et institutionnelle des individus. S'il s'agit de mettre en lumière les dirigeants ayant pris des responsabilités dans des institutions internationales (présidents, secrétaires généraux, membre de comités exécutifs, etc.), les trajectoires commencent presque toujours au niveau national. Dès lors, notre ambition se teinte aussi d'une volonté comparatiste, à la fois entre les individus, les époques peut-être, mais aussi entre les différentes disciplines sportives et les différentes sphères géographiques et politiques (ou idéologiques).

Par ce numéro spécial, nous souhaitons élargir l'horizon des recherches en histoire des relations internationales sportives. Celles-ci rencontrent encore souvent certaines difficultés pour sortir d'un récit qu'on pourrait dire « internaliste ». En effet, les seules formes d'explication et de contextualisation mobilisées relèvent autant d'évolutions, d'hypothèses, d'institutions et/ou de biographies, prises dans le territoire et le temps de la nation. Il convient de préciser que le cadre national est souvent privilégié, car c'est aussi celui du chercheur qui écrit l'histoire. De fait, en raison de la précocité de la mise en place d'institutions internationales sportives, l'objet «sport » permet d'envisager un fructueux dépassement des " frontières nationales ", où les biographies doivent venir densifier notre connaissance des conditions de développement des formes modernes de pratiques corporelles.

L'étude biographique est tout aussi pertinente que les archives que le travail de l'historien cherche à mobiliser. Les nombreux documents analysés ont des origines diverses, qu'ils soient officiels (procès-verbaux de séances institutionnelles), informels ou personnels (lettres, objets ou encore entretiens avec les dirigeants eux-mêmes lorsque c'est encore possible). Ces archives révèlent les motivations profondes et les conceptions des individus autour du sport. Comme le rappelle Jean-Claude Passeron, "l'intelligibilité d'une biographie s'impose [...] d'emblée, en concentrant la transparence fonctionnelle de la "pré-notion", l'évidence existentielle du vécu et l'efficacité dramatique du scénario » (1989, p. 3). Pour autant, comme le rappelle Giovanni Levi dans un article programmatique majeur (paru quelques mois seulement avant l'article de Jean-Claude Passeron), la documentation n'est jamais neutre (1989, p. 1329), et ce sont alors les comparaisons interindividuelles qui permettent d'esquisser un travail plus prosopographique.

Par ailleurs, notre démarche répond aussi à un besoin scientifique et épistémologique. En effet, nous avons pu souligner à de nombreuses reprises (Vonnard \& Quin, 2017 ; Quin \& Vonnard, 2019), voire parfois critiqué et souvent regretté, le manque de connaissance autour de ces acteurs, en particulier leur ancrage social (milieu social, appartenance à des mouvements sociaux ou politiques, cultures politiques, etc.) ou les liens qu'ils ont pu développer entre eux. Un tel travail est à ce jour inédit, il doit permettre une avancée significative dans la compréhension du phénomène sportif et de son histoire. Cela doit aussi permettre de poser les premiers jalons dans l'établissement d'une véritable cartographie des échanges transnationaux entre dirigeants sportifs, qui à ce jour est inexistante.

Ce numéro témoigne d'une volonté de privilégier une approche cumulative de ces biographies de dirigeants internationaux, avec la prise en compte de plusieurs continents (Europe, Afrique, Asie), de plusieurs disciplines sportives (football, ski, cyclisme, etc.), d'approches différentes (biographie individuelle, compréhension de réseaux ou de fonctionnement étatique singulier), mais aussi de contextes spécifiques de l'engagement de ces dirigeants. Ainsi, les contributions se focalisent largement sur les décennies qui suivent la Seconde Guerre mondiale et vont être le 
théâtre d'une accélération de l'internationalisation du sport, voire d'une première globalisation de ces pratiques culturelles, mais les auteurs n'oublient pas de rappeler les racines de ces dynamiques qui remontent à l'entredeux-guerres et de présenter, pour certains, des conséquences plus contemporaines en étendant leurs analyses jusqu'aux années 1980.

La première contribution écrite par Igor Martinache propose une analyse des profils des dirigeants du «sport communiste » en France. Ainsi, il propose de montrer la réciprocité et la complexité des circulations humaines et idéelles dans la sphère du bloc communiste (sphère d'influence soviétique et partis communistes nationaux). Il se donne pour tâche d'éclairer les transformations des positions diplomatiques et doctrinales des organisations concernées (FSGT et le parti communiste français), à partir de l'analyse comparée des trajectoires biographiques d'un certain nombre de dirigeants du «sport communiste » en France à différentes périodes.

Anais Bohuon, Florys Castan Vicente, Pia Henaff-Pineau et Nicolas Chanavat se concentrent de leur côté sur les trajectoires d'Alice Milliat et Marie-Thérèse Eyquem. En effet, très emblématiques, ces pionnières du sport international féminin vont se retrouver contraintes par des idéologies et des pouvoirs conservateurs, et notamment médicaux ou scientifiques. En effet, tout au long de leurs carrières, les deux pionnières vont être confrontées à la sphère médicale, notamment aux discours qui oscillent entre prescriptions et proscriptions au sujet de la mise en mouvement des corps des femmes. Les pratiquantes et organisatrices s'adaptent tant dans le choix et les modalités des activités pratiquées que dans les pratiques de (non-)mixité dans la direction des organisations du sport féminin. Elles évoluent entre transgression et compromis, entre intégration, reproduction ou rejet des normes et injonction à la féminité dans les activités physiques.
Autour de la biographie du dirigeant ghanéen Ohene Djan, Claire Nicolas et Philippe Vonnard proposent de décrypter le rôle d'un acteur majeur du sport africain, tout particulièrement ghanéen, dans les années 1960. Autour d'une trajectoire ascendante, les deux auteurs envisagent d'analyser à la fois la construction d'une politique sportive dans un contexte postcolonial et de comprendre les dynamiques de l'intégration d'un dirigeant extra-européen au sein d'une administration largement gouvernée par des dirigeants occidentaux. Ainsi, entre deux «mondes ", l'ambition de cette contribution est précisément de mesurer les logiques propres d'une trajectoire singulière et, sous certains aspects, pionnière.

Dans sa contribution, Amanda Shuman analyse le rôle des dirigeants sportifs de la République populaire de Chine (RPC) dans la création et le développement de nouveaux réseaux transnationaux entre la création de la RPC en 1949 et le début de la Révolution culturelle en 1966. Au départ très engagée sur la scène internationale sportive, à la fois occidentale (avec les Jeux olympiques) et dans le cadre d'événements comme les GANEFO, plus spécifiques à un contexte de décolonisation et d'essor du sport dans la sphère socialiste, la Chine veut se positionner comme un acteur majeur entre les États-Unis, l'Europe et l'URSS. Au courant des années 1960, avec la Révolution culturelle, la Chine va rapidement se retirer de la scène internationale et va notamment transformer fortement la gouvernance de son système sportif. Amanda Shuman souligne encore qu'il faudra ensuite attendre la mort de Mao et la fin de la révolution culturelle pour que les nouveaux dirigeants chinois décident de revenir au CIO dans le cadre du plan visant à (re)positionner la Chine sur la scène mondiale comme « ouverte ", " réformée » et «modernisée ».

Dans leur contribution, Sébastien Cala et Grégory Quin se donnent pour tâche de décrire l'avènement de la modalité "alpine » du ski - traditionnellement dominé par les 
dirigeants (et les pays) nordiques jusqu'aux premières décennies du $\mathrm{XX}^{\mathrm{e}}$ siècle - dont la gouvernance revient à des dirigeants suisses. Si la carrière de Marc Hodler est emblématique, avec notamment 47 ans de présidence de la FIS, elle révèle aussi la densité des réseaux qui existent autour du ski (et des sports d'hiver). De fait, ces réseaux dépassent largement la sphère sportive, ils s'articulent et s'enracinent dans les dynamiques des industries touristiques et hôtelières, et ils sont affectés par les soubresauts d'un contexte géopolitique singulier entre 1920 et 1960.

L'historien italien Nicola Sbetti propose de son côté une analyse de la trajectoire d'Alberto Bonacossa. Dirigeant emblématique qui fut tour à tour président de la Fédération internationale de motocyclisme, vice-président de celle des Automobile-clubs, membre de la Ligue internationale de hockey sur glace et de la Fédération internationale de patinage, mais surtout, à partir de 1925, membre du CIO et quelques années plus tard (dès 1935) membre actif de son comité exécutif. Dans son article, Nicola Sbetti ambitionne d'analyser en détail les étapes de la carrière d'Alberto Bonacossa, en particulier pour mettre en lumière l'importance de ses racines aristocrates dans le cadre de son engagement dans des réseaux sportifs internationaux dominés par une culture à la fois cosmopolite, transnationale et patriotique/nationaliste. Cette contribution repose notamment sur un recueil colossal d'archives provenant du comité olympique national italien (CONI) et du CIO. Ces dernières permettent de souligner la manière dont les tensions entre le national et l'international se réactualisent singulièrement au sein des organisations sportives internationales.

Dans sa contribution centrée sur les réseaux sportifs de la ville de Reims (des années 1900 aux années 1960), Sébastien Moreau s'attache à remettre en perspective les trajectoires de trois dirigeants majeurs de la scène internationale sportive dans la première moitié du XX $\mathrm{XX}^{\mathrm{e}}$ siècle, à savoir Louis Daugé, Teddy Kriegk et Melchior de Polignac. À l'aide d'une prosopographie nourrie de travaux d'histoire sociale et institutionnelle, cette démarche permet d'évaluer les dynamiques de démocratisation, d'institutionnalisation et de hiérarchisation du monde sportif, et leurs effets sur la vie sportive locale et la composition du groupe des présidents de clubs. La nomination de dirigeants fédéraux régionaux est à ce titre un processus clé pour comprendre les processus singuliers de l'imposition de l'autorité fédérale sur les pratiques sportives et l'articulation d'un tissu sportif local (et ses présidents de clubs) à l'édifice institutionnel international (et ses plus hauts dirigeants).

L'entretien réalisé par Grégory Quin avec l'historien britannique Richard Holt permet de revenir sur la trajectoire d'un acteur majeur de l'historiographie britannique et internationale de ces quarante dernières années, dont l'influence sur l'histoire du sport est appelée à durer. En outre, cet entretien est une occasion unique de relire quarante années de l'histoire du sport, où les opportunités de collaborations internationales sont souvent remises en question par des carcans nationaux très puissants. D'occasions manquées en réels besoins d'échanges, cet entretien justifie aussi la production de projets internationaux de recherche, ce dont témoigne aussi la réalisation de ce numéro spécial.

Enfin, dans leur article, Philippe Vonnard, Gil Mayencourt et Hans-Dieter Gerber souhaitent présenter les cadres méthodologiques et leurs premiers résultats d'un projet de recherche sur les élites du sport suisse au tournant du XXe siècle. Le projet intitulé « La Fabrique des sports nationaux "- financé pour la période 2019-2023 par le Fonds national suisse pour la recherche scientifique (FNS) - propose de se concentrer sur la genèse et les premiers développements des sports en Suisse de 1860 à 1930. Ce processus d'établissement des principaux sports dans la société helvétique suit plus généralement le développement politique, économique et culturel du pays 
lui-même, dont la structure étatique moderne date de 1848. La recherche porte sur le développement de cinq sports : le cyclisme, la gymnastique, le football, le tir et ce que l'on appelle généralement les " Jeux nationaux " constitués par le hornuss et la lutte suisse, dont l'essor doit aussi se comprendre dans un contexte plus large d'internationalisation de la pratique et de la participation des dirigeants helvétiques à la mise en place des premières organisations internationales sportives dès les années 1880 .

Les contributions de ce numéro spécial sont en outre issues de nombreux échanges ayant eu lieu dans les dernières conférences du RERIS (Réseau d'Etude des Relations Internationales Sportives).

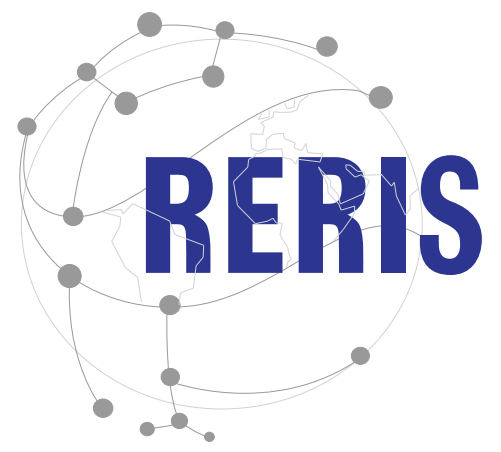

\section{BibLIOGRAPHIE}

Bayle, E. (2014). Les grands dirigeants du sport. 23 portraits et stratégies de management. Bruxelles: De Boeck.

Bourdieu, P. (1986). L'illusion biographique. Actes de la recherche en sciences sociales, 62-63, 69-72.

Carter, N. (2006). The football manager, a history. London: Routledge.

Clastres, P. (2005). Inventer une élite : Pierre de Coubertin et la « chevalerie sportive ", Revue française des idées politiques, 22(2), 51-71.

Clastres, P. (2014). Cultures sportives et cultures politiques. Le cas des chefs d'État et de gouvernement dans le monde au $\mathrm{XX}^{\mathrm{e}}$ siècle. Histoire@Politique, 23(2), en ligne.

Dyer, L. \& Day, D. (2017). The Industrial middle class and the development of sport in a railway town. Sport in History, 37(2), 164-182.

Gerber, H.-D. (2007). Fussball in Basel von den Anfängen bis zum Zweiten Weltkrieg. Basler Zeitschrift für Geschichte und Altertumskunde, 107, 7-33.

Homburg, H. (2007). Ernst Thommen, die Schweiz und der Weltfussball, 1946-1962, Basler Zeitschrift für Geschichte und Altertumskunde, 107, 69-102.

Lemercier, C. \& Picard, E. (2012). Quelle approche prosopographique? In P. Nabonnand \& L. Rollet (dir.), Les uns et les autres... Biographies et prosopographies en histoire des sciences (pp. 605-630). Nancy : Presses universitaires de Nancy.

Levi, G. (1989). Les usages de la biographie. Annales E.S.C., 44(6), 1325-1336.

MacAloon, J. (2008). This great symbol: Pierre de Coubertin and the origins of the modern Olympic Games. London: Routledge.

Milza, P. (2012). Figures de grands décideurs : l'intérêt de la biographie. In R. Frank (dir.), Pour l'histoire des relations internationales (pp. 545-559). Paris : Presses universitaires de France.

Passeron, J.-C. (1989). Biographies, flux, itinéraires, trajectoires. Revue française de sociologie, 31(1), 3-22.

Peiffer, L. \& Schulze-Marmeling, D. (dir.) (2008). Hakenkreuz und rundes Leder. Fussball im Nationalsozialismus, Göttingen: Die Werkstatt.

Pennetier, C. et al. (2006). Dictionnaire biographique, mouvement ouvrier, mouvement social : période 19401968, de la Seconde Guerre mondiale à mai 1968. Paris : Les Éditions de l'Atelier/Les Éditions ouvrières.

Quin, G. \& Vonnard, P. (2019). Promouvoir l'internationalisation du football : les Dirigeants suisses et la Fédération Internationale de Football Association (FIFA) (1904-1954). Storia dello sport, 1(1), 4-22.

Quin, G., Vonnard, P. \& Jaccoud, C. (2019). Des réseaux et des hommes. Participation et contribution de la Suisse à linternationalisation du sport (1912-1972). Neuchâtel : Alphil. 
Szerdahelyi, L. \& Robène, L. (dir.) (2019). Les femmes, actrices de l'EPS. Staps, 123(1).

Tomlinson, A. (2000). FIFA and the men who made it. Soccer E Society, 1(1), 55-71.

Vramplew, V. (2016). Playing together : towards a theory of the British sports club in history. Sport in Society, 19(3), 455-469.

Vonnard, P. \& Quin, G. (2017). Did South America foster European football ? : Transnational Influences on the Continentalisation of FIFA and the Creation of UEFA, 1926-1959. Sport in Society, 20(10), 1424-1439. 